\title{
Separation of catecholamines by microchip electrophoresis with a simple integrated laser-induced fluorescence detector
}

\author{
Hai-Fang $\mathrm{Li}^{\mathrm{a}}$, ZongWei Cai ${ }^{\mathrm{b}}$, Jin-Ming Lin ${ }^{\mathrm{a}, \mathrm{c}, *}$ \\ a Department of Chemistry, Tsinghua University, Beijing 100084, China \\ ${ }^{\mathrm{b}}$ Department Chemistry, Hong Kong Baptist University, Kowloon Tong, Hong Kong, China \\ c State Key Laboratory of Environmental Chemistry and Ecotoxicology, Research Center for Eco-Environmental Sciences, \\ Chinese Academy of Sciences, P.O. Box 2871, Beijing 100085, China
}

Received 6 December 2005; received in revised form 13 February 2006; accepted 13 February 2006

Available online 28 February 2006

\begin{abstract}
A simple microchip electrophoresis-laser-induced fluorescence device was constructed and used for separation and determination of catecholamines. On the fabricated glass chip, an extra optical fiber insertion channel, which was perpendicular and extremely close to the separation channel, was directly integrated by nothing operations more than design features on the photomask. The utilization of optical fiber to transmit the excitation light and the integration fiber channel make the fluorescence detection system simple and disposable. For electrophoresis, optimization of separation conditions was investigated for reaching high separation efficiency and sensitivity. A separation efficiency as high as $10^{6}$ theoretical plate numbers could be obtained for the analytes.
\end{abstract}

(C) 2006 Elsevier B.V. All rights reserved.

Keywords: Microchip electrophoresis; Laser-induced fluorescence; Optical fiber; Catecholamines

\section{Introduction}

The microchip capillary electrophoresis (MCE) originally pioneered by Harrison et al. [1] have been rapidly developed in recent years [2]. Compared with conventional capillary electrophoresis (CE), MCE exhibits special potential advantages including rapid analysis, reduced sample and reagent consumption, integration and disposability [3]. To realize sensitive analysis of various samples, many conventional detection schemes such as absorbance, fluorescence, chemiluminescence, electrochemical, electrochemiluminescence, and mass spectroscopy have been employed in MCE [4]. Among these applications, laser-induced fluorescence (LIF) and electrochemical (EC) are the most popular detection techniques for MCE devices [5,6]. EC detection technique for MCE has been mainly focused on detection of catechols [7], phenols [8], and nitroaromatics [9]. To date, EC detection methods used for $\mathrm{CE}$ or MCE can be divided into three main categories, i.e., amperometry, conductivity and

\footnotetext{
* Corresponding author. Tel.: +86 1062792343 ; fax: +86 1062841953 . E-mail address: jmlin@ mail.tsinghua.edu.cn (J.-M. Lin).
}

potentiometry [6]. While some successes have been realized, implementing EC detection on chip may be extremely difficult and tedious in terms of electrodes preparation and integration involve complicated fabrication and handling procedures. Furthermore, some instrumental considerations with microchip EC detection must be taken account of in comparison to optically based detection methods. For example, since the detection reservoir contains the working, anxiliary, and reference electrodes connected to a potentiostat, it must always be held at ground. It has been proposed that those separations requiring reverse separation (anode detection) can't be performed. For the amperometric detection, when the separation channel is wider than $25 \mu \mathrm{m}$, a decoupler is needed to eliminate the separation voltage interference in EC current [10]. The conductivity detections on chip also encounter problems. The hydrolysis of water and gas bubble formation would take place at the electrode surface at high electrophoresis field strength, for example, above $100 \mathrm{~V} \mathrm{~cm}^{-1}$ [11]. In addition, the alignment between EC electrodes with the end pre-separation channel, and the repetitious cleaning to activate the EC electrodes between runs complicate the overall analytical procedure. In contrast, these considerations and complicates are not involved in microchip optical 
detection methods. For example, when the channel producing low electro-osmotic flow (EOF) and detecting anions, anode detection, viz. voltages can be applied to the detection reservoir without affecting the detector response. As compared with EC, LIF detection may appear more sensitive, disposable and compatible for on-line detection on chip. The excitation beam can be easily focused and illuminate a small region of the separation channel. Furthermore, diversified wavelengths light emitting diodes and laser diodes can be used as miniaturized excitation sources replacing bulk conventional lasers to realize on-line miniaturized detection $[12,13]$. There are no doubts that the on-line detection mode is preferred to off-line detection mode for realization of integration and high sensitivity of the microdevices.

Dopamine (DA) and epinephrine (E) play a major role as neurotransmitters in central and peripheral nervous systems $[14,15]$. Much interest has been focused not only on researching the bioactive function of these catecholamines but also on developing the selective methods for their detection in biological systems. Although neurotransmitters are normally detected electrochemically, LIF detection has been reported in CE with isofluorothiocyanate (FITC) derivatization to increase detection sensitivity $[16,17]$.

In this study, a simple LIF detection configuration was developed for MCE and applied to determine catecholamines. The present system features the simplest device construction in principle. A blue diode laser was used as excitation source whose output was focused into an optical fiber to remotely illuminate a small region on separation channel. To the best of our knowledge, this is the first report of FITC derivatization of catecholamines to carry out high sensitive fluorescent detection in MCE system. The basic properties of the analytical system such as separation efficiency, detection sensitivity and reproducibility, as well as some interesting findings were demonstrated. Under the optimum separation conditions, the quantification of E in epinephrine hydrochloridum injection and DA in dopamine hydrochloridum injection was determined, respectively. Furthermore, the method was applied for measurements of catecholamines in a spiked urine sample.

\section{Experimental}

\subsection{Chemicals and solutions}

All chemicals were of analytical grade and were used without further purification in our experiments. The derivatizing agent FITC, and standard epinephrine hydrochcloride were purchased from Sigma-Aldrich (St. Louis, MO, USA). Standard dopamine hydrochloride was obtained from Fluka (Buchs, Switzerland). Epinephrine hydrochloride injection $\left(1 \mathrm{mg} \mathrm{mL}^{-1}\right)$ and dopamine hydrochloride injection $\left(10 \mathrm{mg} \mathrm{mL}^{-1}\right)$ were obtained from a local hospital. The $10 \mathrm{mM} \mathrm{Na} \mathrm{B}_{4} \mathrm{O}_{7} / \mathrm{HCl}$ solution was used as electrophoretic buffer and the $\mathrm{pH}$ value was adjusted to $8.0-10.0$ by adding $\mathrm{HCl}$ or $\mathrm{NaOH}$ solution. Stock solutions of $10 \mathrm{mM}$ E and DA were prepared in $10 \mathrm{mM}$ $\mathrm{Na}_{2} \mathrm{~B}_{4} \mathrm{O}_{7} / \mathrm{HCl}$ ( $\mathrm{pH}$ 9.2) buffer. The $10 \mathrm{mM}$ FITC labeling reagent was prepared in acetone with $1 \%(\mathrm{v} / \mathrm{v})$ pyridine added.
All solutions were filtered through $0.25 \mu \mathrm{m}$ membrane filters prior to use.

\subsection{Derivatization procedure}

Derivatization of catecholamines with FITC was realized according to the procedures described previously [18]. Individual FITC-labeled E and DA solution was prepared by mixing $0.1 \mathrm{~mL}$ stock solution of $\mathrm{E}$ or DA with $0.5 \mathrm{~mL}$ of FITC reagent and diluting to $1.0 \mathrm{~mL}$ with $10 \mathrm{mM} \mathrm{Na}_{2} \mathrm{~B}_{4} \mathrm{O}_{7} / \mathrm{HCl}(\mathrm{pH} 9.2)$ buffer, and allowing to stand overnight in the dark at room temperature. The individual FITC-labeled E and DA solutions was firstly analyzed by electrophoresis separation to identify the derivatization. Once the derivatization was utilized, the labeled solutions were stored at $4{ }^{\circ} \mathrm{C}$. A sample solution of $25 \mu \mathrm{M}$ FITClabeled $\mathrm{E}$ and DA was prepared by mixing the labeled stock solutions and diluted with the $10 \mathrm{mM} \mathrm{Na}_{2} \mathrm{~B}_{4} \mathrm{O}_{7} / \mathrm{HCl}$ buffer. Because of the instability of FITC, the sample solution should not be reserved at the room temperature for a long time.

\subsection{Real samples preparation and derivatization}

The derivatization of injection samples and spiked urine was same to the above. The labelling sample solutions were diluted with the $\mathrm{Na}_{2} \mathrm{~B}_{4} \mathrm{O}_{7} / \mathrm{HCl}$ ( $\mathrm{pH}$ 9.2) buffer for a basic media. The epinephrine and dopamine hydrochloride injections were diluted before use without further purification. Finally, a $15 \mu \mathrm{gL}^{-1}$ FITC-labeled epinephrine hydrochloride injection sample and a $38 \mu \mathrm{g} \mathrm{L}^{-1}$ FITC-labeled dopamine hydrochloride injection sample were obtained.

\subsection{Fabrication of microchip}

With the standard photolithography and wet chemical etching techniques [19], the microchip was fabricated from a $6.3 \mathrm{~cm} \times 6.3 \mathrm{~cm}$ soda lime glass substrate precoated with $145 \mathrm{~nm}$ Cr layer and $570 \mathrm{~nm}$ positive AZ1518 photoresist (Shaoguang Microelectronics Corp., Changsha, China). An imprinted photomask as shown in Fig. 1a was designed, on which there are two identical optical fiber (OP) insertion channels except of the microfluidic channels, i.e., the sample loading channel and the separation channel. The channel structures on the photomask were transferred onto the glass substrate following an UV exposure. The OP insertion channels were developed in a $0.5 \%(\mathrm{w} / \mathrm{w})$ $\mathrm{NaOH}$ solution but the microfluidic channels were shielded with a waterproof adhesive tape. The deep OP insertion channels were etched on the glass substrate in a stirred $1.0 \mathrm{M} \mathrm{HF} / \mathrm{NH}_{4} \mathrm{~F}$ etchant. When the dimension of the OP insertion channels reached the expected depth of $85 \mu \mathrm{m}$, the glass substrate was removed from the etchant and washed clearly. Then the adhesive tape microfluidic channels were tore off and the remainder was cleaned with the ethanol, and the microfluidic channels were developed in the $\mathrm{NaOH}$ solution. After covering the OP insertion channels area with the waterproof adhesive tape, the glass substrate was etched in a $0.3 \mathrm{M} \mathrm{HF} / \mathrm{NH}_{4} \mathrm{~F}$ etchant. When the etching process was accomplished, the glass substrate was departed from the axis into two parts serving as the top and bottom plates of chip. 


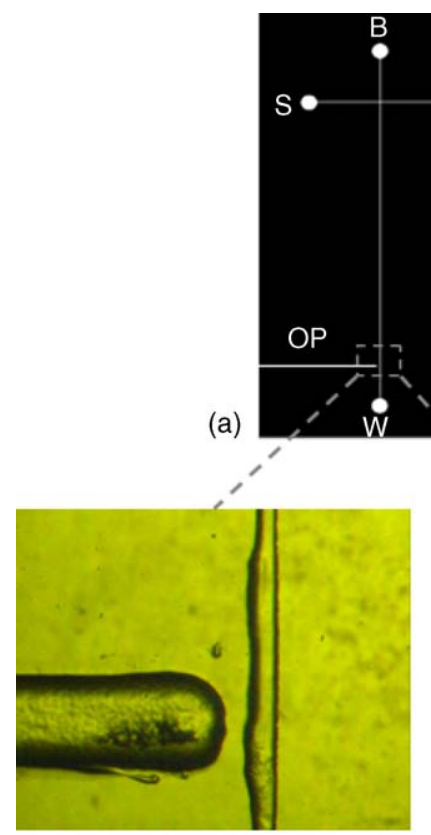

(b) a depth of $170 \mu \mathrm{m}$, and which end is $190 \mu \mathrm{m}$ away from the separation channel.

\subsection{LIF detection}

As illustrated in Fig. 2, a simple MCE-LIF detection system with the virtue of optical fiber was constructed and some characteristics of the system have been demonstrated in detail previously [20]. A $473 \mathrm{~nm}$ blue diode laser (Beijing Viasho Technology, China) was used as the excitation source. The laser beam was focused into a multimode optical fiber $(100 \mu \mathrm{m}$ core diameter and $140 \mu \mathrm{m}$ cladding diameter), the other tip of which was inserted into the OP channel on the chip. The laser beam perpendicularly illuminated on the separation channel. Fluorescence was directly detected by a photomultiplier tube after passing through a $400 \mu \mathrm{m}$ diameter pinhole and optical filters of holographic notch plus filter $(476 \mathrm{~nm})$ and interference filter $(535 \mathrm{~nm})$. A CDY-800L high-voltage switching apparatus which consists of four $8000 \mathrm{~V}$ high-voltage power supplies (Shandong Chemical Engineering Institute, China) was used for electrophoresis.

\subsection{Microchip electrophoresis}

Prior to electrophoresis, the microfluidic channels were flushed sequentially with $1 \mathrm{M} \mathrm{NaOH}$ for $10 \mathrm{~min}$, deionized water for $5 \mathrm{~min}$ and the separation electrolyte for $10 \mathrm{~min}$. The $10 \mathrm{mM}$ $\mathrm{Na}_{2} \mathrm{~B}_{4} \mathrm{O}_{7} / \mathrm{HCl}$ solutions with different $\mathrm{pH}$ value were served as running buffer for the capillary zone electrophoresis separation. Before repetitive runs started, sample plug formation was simply achieved by use of a cross-shaped injector as follows. The sample was electrokinetically delivered from the sample reservoir (S) to the sample waste reservoir (SW) by applying $900 \mathrm{~V}$ to $\mathrm{S}$ reservoir, with SW reservoir held at ground. Different sample loading times were attempted. When a high potential was applied to the buffer reservoir (B) with the buffer waste reservoir (W) grounded, the sample plug of the intersection was injected into and migrated down along the separation channel. During separation back voltages were applied to $S$ and of the separation channel. The unitary OP insertion channel has

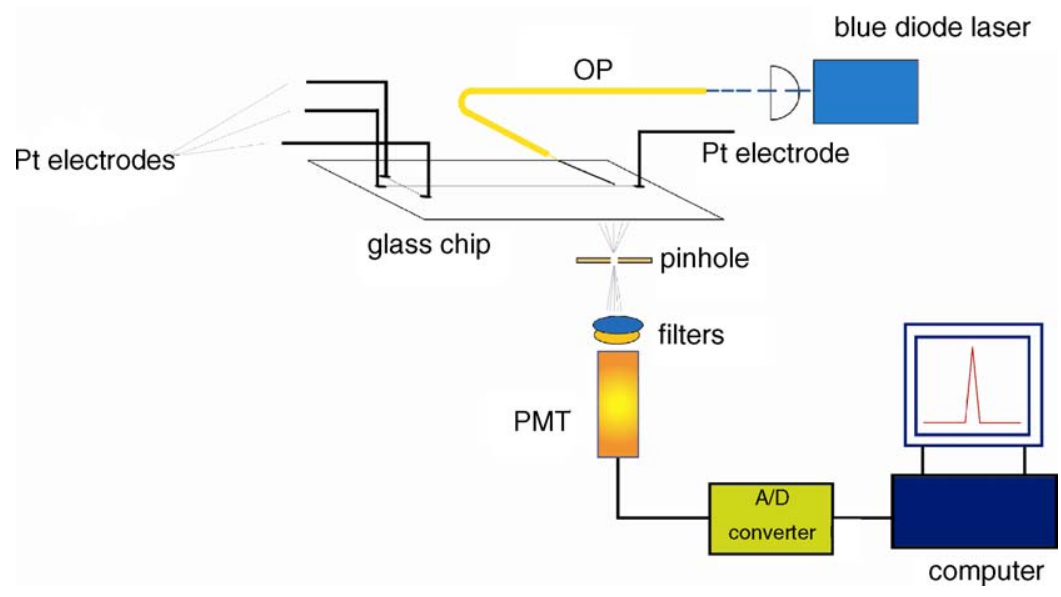

Fig. 2. The home made MCE-LIF detection system. 
SW reservoirs. The separation voltage was changed from 1500 to $3500 \mathrm{~V}$.

\section{Results and discussion}

\subsection{Characterization of the integrated MCE-LIF chip}

The MCE-LIF chip used in the present work featured an optical fiber channel integrated on the chip for insertion of the optical fiber, so that no extra optics are required to fix and align the optical fiber with the detection point. In order to make the optical fiber core horizontal to the axis of the separation channel, the OP insertion channel was separately designed in the top and bottom plates. According to the separation channel depth and the $140 \mu \mathrm{m}$ cladding diameter of OP, the dimensions of the two OP insertion channels on the plates were etched to about $85 \mu \mathrm{m}$ deep and $200 \mu \mathrm{m}$ average wide by controlling the etching time. Owing to that the designed OP insertion channel was exactly perpendicular to the separation channel and the depth of the unitary OP insertion channel just accorded to the cladding diameter of the optical fiber, the optical fiber could be self-aligned to the detection point once it was inserted into the OP insertion channel.

To reduce the dispersion of the excitation light, the distance between the end of the OP insertion channel and the separation channel should be as short as it could be controlled. In our previous experiments as reported [20], if the separation channel was etched prior to further deep etching the OP insertion channel, as shown in Fig. 1b, the detection region, viz. the region profile of the separation channel near the end of the OP insertion channel was easily distorted when the short distance between them was desired. In contrast, if we etched the separation channel after completing fabrication of the OP insertion channel, the detection region could be protected perfectly (shown in Fig. 1c), The minimum distance between the separation channel and the end of the OP insertion channel was controlled to be as short as $190 \mu \mathrm{m}$. According to the calculational methods described previously [20], the excitation beam launched from the optical fiber with $100 \mu \mathrm{m}$ core diameter will give an expanded laser spot of $156 \mu \mathrm{m}$ diameter on the separation channel. This excitation spot was small enough for the MCE separation. The strategies of using the optical fiber to transmit the excitation light and integrating OP insertion channel on chip make the alignment and coupling between excitation source and the detection point simple and disposable without any extra optics.

\subsection{Effect of the pH of buffer on derivatization and separation}

According to the previous reports [18,21], excellent FITC derivatization could be achieved under weak alkaline conditions. This is because that the amino groups of the catecholamines would easily be deprotonated in alkaline buffer and the FITC fluorescent intensity in alkaline buffer rapidly increased. In our experiments, it was found that when the $\mathrm{pH}$ of the derivatization buffer was around 9.0, the fluorescence signal of FITC-DA was

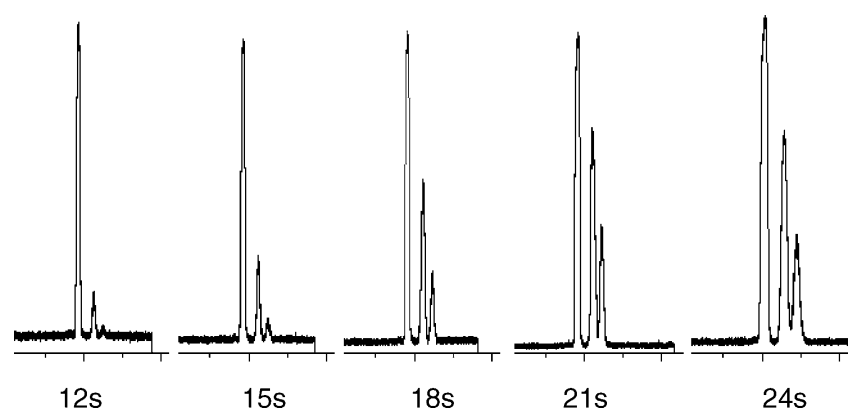

Fig. 3. Effects of the sample loading time on sensitivity. The loading times increase from 12 to $24 \mathrm{~s}$ in $3 \mathrm{~s}$ steps. Conditions: sample loading voltage, $900 \mathrm{~V}$; sample containing $25 \mu \mathrm{M}$ FITC-labeled E and DA.

intensive, so the buffer of $10 \mathrm{mM} \mathrm{Na}_{2} \mathrm{~B}_{4} \mathrm{O}_{7} / \mathrm{HCl}(\mathrm{pH} 9.2)$ was used to dilute the derivatization solution.

Since the fluorescein group is highly fluorescent in the alkaline medium, the optimization of $\mathrm{pH}$ value of the electrophoretic buffer was just carried out in the 8.0-10.0 range in 0.4 steps. According to the peak resolution and migration time differences between FITC-E and FITC-DA in electropherograms, it was found that, when the buffer $\mathrm{pH}$ was at 9.2, the separation of FITC-E and FITC-DA was slightly better than at other $\mathrm{pH}$ values. Therefore, the $\mathrm{pH}$ value of 9.2 was selected for the electrophoretic buffer.

\subsection{Effect of sample loading time on detection sensitivity}

Because of the short separation channel on chip, the sample injection plug has a very high influence on the separation results. It has been proved that electric injection mode easily induces bias between analytes and leads to signal peaks distorting the primal sample composition. Increasing sample loading time could reduce influence of bias at some extent. However, for the floating injection mode on a cross channel structure, there is a sample diffusion phenomenon towards the separation channel when just adding a potential on the injection channel. The longer sample loading time leads to the fiercer diffusion. Therefore, optimization sample injection procedure is necessary to reduce the bias and diffusion of analytes.

In our experiments, the influence of sample loading time on separation was valued. Under the sample loading potential of $900 \mathrm{~V}$, the loading time was changed from $12,15,18,21$ to $24 \mathrm{~s}$. The repetitive separation runs were performed under the same separation conditions. As can be seen in Fig. 3, with prolonging the sample loading time from 12 to $21 \mathrm{~s}$, while the FITC peak height almost kept a constant value, the peaks height of FITC-E and FITC-DA increase markedly. This illustrates that the longer loading time could reduce the bias in sample plug. Further increase in sample loading time up to $24 \mathrm{~s}$ no longer improved signals of all analytes but caused band-broadening, which indicated that the diffusion was serious and theoretical plates decreased. Finally, the sample loading time of $21 \mathrm{~s}$ was selected as the optimal sample loading time by considering the separation efficiency and the sensitivity. In the following experiments, the floating sample loading operation of $900 \mathrm{~V}$ for $21 \mathrm{~s}$ was performed unless otherwise specified. 
Table 1

Effects of applied voltages during separation on the migration times, theoretical plates and the minimum resolution

\begin{tabular}{|c|c|c|c|c|}
\hline \multicolumn{2}{|l|}{ Applied voltages (V) } & \multirow[t]{2}{*}{ Separation time (s) } & \multirow[t]{2}{*}{ Minimum resolution } & \multirow[t]{2}{*}{ Theoretical plates } \\
\hline Separation voltage & Back voltage & & & \\
\hline 1500 & 450 & 92 & - & 37322 \\
\hline 2000 & 700 & 52 & 1.28 & 82234 \\
\hline 2500 & 900 & 41 & 1.75 & 127734 \\
\hline 3000 & 1100 & 31 & 2.0 & 114298 \\
\hline 3500 & 1300 & 27 & - & 27420 \\
\hline
\end{tabular}

\subsection{Electrophoresis separation}

Separation voltage was an important parameter in CE especially in MCE. Since the separation channel on $\mathrm{cm}^{2}$ scale chip was just several centimeters long, the effect of separation voltage on separation speed and efficiency was distinct. As previous reported [22], during separation just controlling of the potential at the buffer and buffer waste reservoirs led to sample leakage from the sample injection channel. To alleviate the leakage, in the separation phase adding appropriate back voltages at the sample reservoir and the sample waste reservoir was necessary. Therefore, in our experiments, after the same sample loading operations, the separation voltage and the back voltages were simultaneously adjusted. The effects of separation voltages on the separation time, theoretical plate numbers (FITC as representation), and the minimum resolutions (between FITCE and FITC-DA) were listed in Table 1. As the separation voltage increased, the separation time decreased, the resolution improved, and the theoretical plate numbers increased. At the same time, when the separation voltage was too high, the migration time differences of analytes would be reduced, the resolution became poor and the theoretical plate numbers rapidly decreased. These may be caused by the diffusion resulting from Joule heat induced by high eletrophoretic current. A representative electropherogram of FITC derivatives and untreated labeling agent is shown in Fig. 4, demonstrating the excellent separation of these analytes under the separation voltage of $3000 \mathrm{~V}$. For the FITC, FITC-E and FITC-DA, the half-width of peak was as narrower as $0.9,1.0$ and $1.0 \mathrm{~s}$, respectively, and so the theoretical plate numbers of the analytes peaks are up to the order of $10^{6}$. Under the optimal sample loading and separation conditions, a set of standard sample solutions were tested and the concentrations ranging from $2.0 \times 10^{-4}$ to $1.7 \times 10^{-7}$ and $2.0 \times 10^{-4}$ to $1.0 \times 10^{-7} \mathrm{M}$ for DA and $\mathrm{E}$, respectively, could keep linear. The detection limits for DA and $\mathrm{E}$ are 0.05 and $0.01 \mu \mathrm{M}$, respectively.

\subsection{Reproducibility}

Separation reproducibility is crucial for a practical attractiveness of an analytical system. In MCE-EC analysis, EC current and the shift in half-wave potential $[23,24]$ of analytes are affected by the relative position of the working electrode to the separation channel outlet. The relative variations of EC current was inevitably caused by the easily contaminated working electrodes. Therefore, the fixation and activation of the working electrode between analysis runs are necessary for keeping reproducibility. In contrast, LIF detection sections, no requirements of directly contacting with the sample and buffer solutions, are easy to keep good reproducibility. In our experiments, with the optimum sample loading and separation conditions, replicates of $25 \mu \mathrm{M}$ FITC-labeled E and DA sample were performed. Both the migration times and the fluorescence signals of analytes for the seven runs were reproducible. For the analytes of FITC, FITC-E and FITC-DA, relative standard deviations (RSDs) of migration times were $2.9,3.5$ and $3.8 \%$, respectively, and RSDs of peak heights signal were $2.7,3.1$ and $4.2 \%$, respectively.

\subsection{The application}

This simple MCE-LIF system was applied to quantifying the epinephrine and dopamine hydrochloridum injections, and analyzing the spiked urine sample. Under the optimum separation conditions, the representative electropherograms of the $15 \mu \mathrm{g} \mathrm{L}^{-1}$ FITC-labeled epinephrine hydrochloride injection

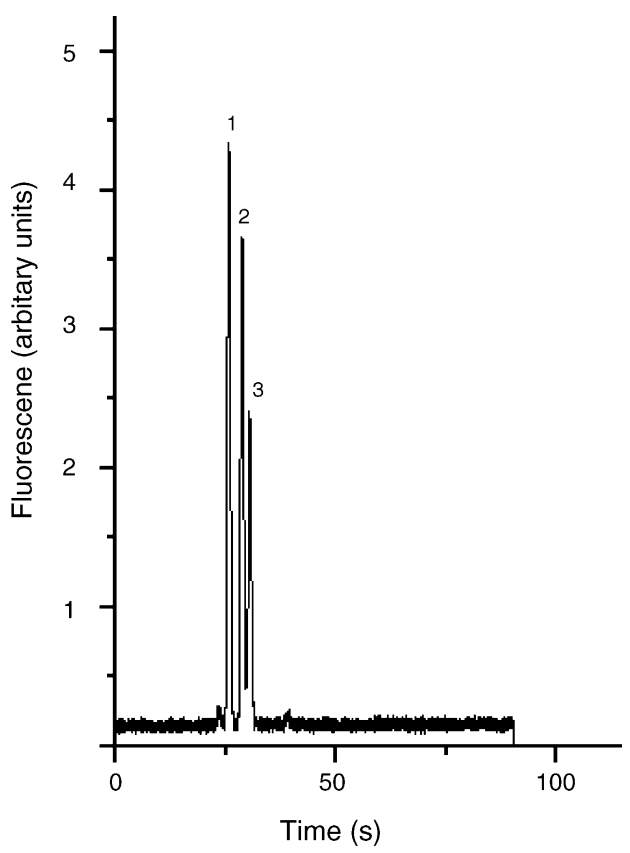

Fig. 4. Typical electropherogram of separation of $25 \mu \mathrm{M}$ FITC-labeled DA and E. Analytical conditions: $10 \mathrm{mM} \mathrm{Na}_{2} \mathrm{~B}_{4} \mathrm{O}_{7} / \mathrm{HCl}$ ( $\mathrm{pH}$ 9.2) running buffer; the floating sample loading operation of $900 \mathrm{~V}$ for $21 \mathrm{~s}$; the separation voltage of $3000 \mathrm{~V}$ and back voltage of $1100 \mathrm{~V}$. Peaks: 1, FITC; 2, FITC-E; 3, FITC-DA. 


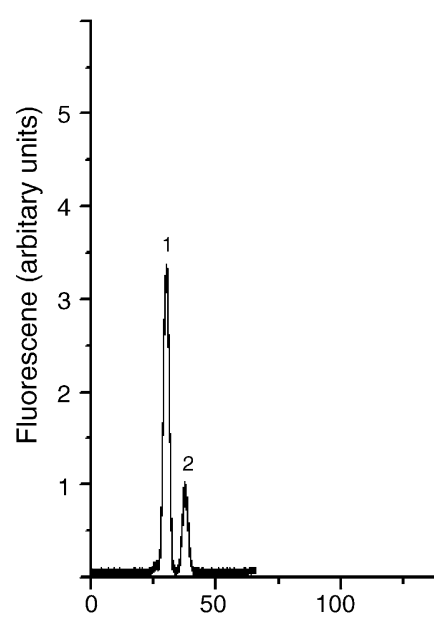

(a)

Time (s)

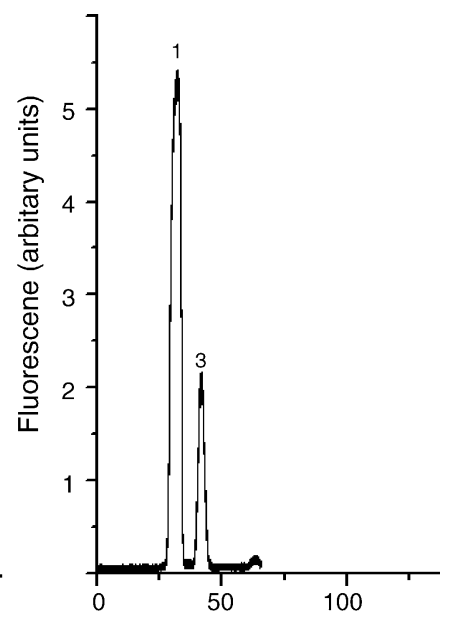

(b) Time (s)

Fig. 5. Electropherogram of samples. (a) $15 \mu \mathrm{g} \mathrm{L}^{-1}$ FITC-labeled epinephrine hydrochloride injection sample. (b) $38 \mu \mathrm{g} \mathrm{L}^{-1}$ FITC-labeled dopamine hydrochloride injection sample. For analytical conditions see Fig. 4.

sample and the $38 \mu \mathrm{g} \mathrm{L}^{-1}$ FITC-labeled dopamine hydrochloride injection sample were obtained as shown in Fig. 5. According to the linearity range of the analytes, the calculated result contents were $0.93 \mathrm{mg} \mathrm{mL}^{-1}$ of $\mathrm{E}-\mathrm{HCl}$ in epinephrine hydrochloridum injection and $10.47 \mathrm{mg} \mathrm{mL}^{-1}$ for DA-HCl in dopamine hydrochloridum injection, respectively. The recoveries were tested by spiked standard E or DA diluted solutions into its corresponding hydrochloridum injections, and the results were 90 and $93 \%$ for epinephrine and dopamine hydrochloridum injections, respectively. Fig. 6 showed the electropherogram of

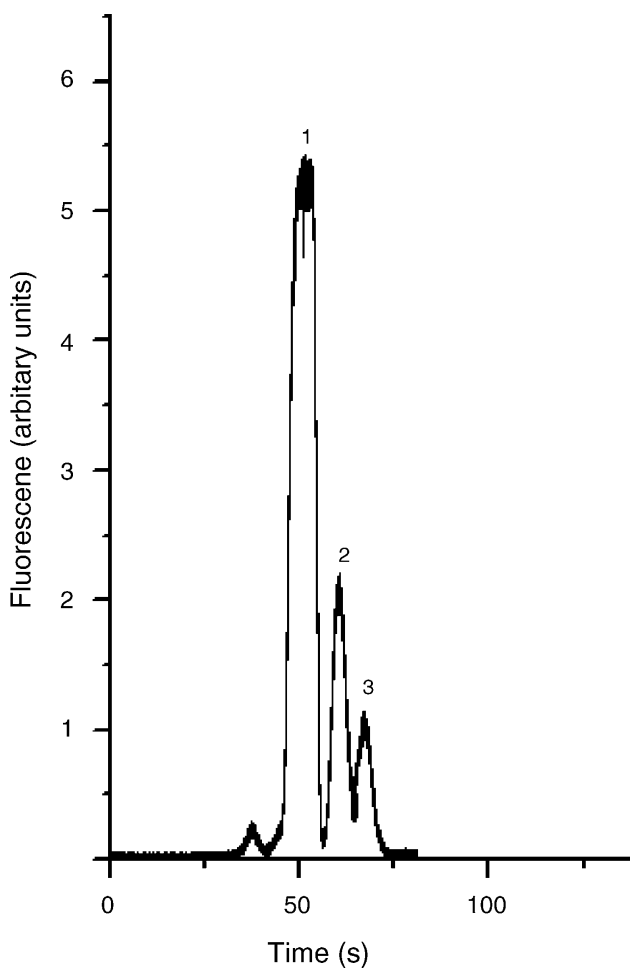

Fig. 6. Electropherogram of the urine sample spiked with $15 \mu \mathrm{M}$ E and DA. Peaks: 1, FITC; 2, FITC-E; 3, FITC-DA. For analytical conditions see Fig. 4.

the spiked urine sample with standard $15 \mu \mathrm{M} \mathrm{E}$ and DA. The recoveries of $\mathrm{E}$ and DA were calculated to be 109 and $87 \%$, respectively.

\section{Concluding remarks}

A simple MCE-LIF detection system has been developed for the separation and detection of E and DA after FITC derivatization. Several operational parameters, such as sample loading time, the separation voltages, have been investigated to obtain a better separation result and better peak signals of components. The results indicate that the described method is simple and well suited for performing sensitive and reproducible determination of neurotransmitters.

As compared to previously published microanalysis neurotransmitters on chip, the present method is the first performance of derivatization of neurotransmitters to carry out fluorescence detection in MCE.

Finally, one can point out that the present MCE-LIF detection system could be easily operated and friendly used by anyone.

\section{Acknowledgements}

The authors gratefully acknowledge financial support of National Natural Science Foundation of China (nos. 20437020 and 20575008).

\section{References}

[1] D.J. Harrison, A. Manz, Z. Fan, H. Luedi, H.M. Widmer, Anal. Chem. 64 (1992) 1926.

[2] V. Dolník, S. Liu, S. Jovanovich, Electrophoresis 21 (2000) 41.

[3] M. Freemantle, Chem. Eng. News 77 (1999) 27.

[4] K.B. Mogensen, H. Klank, J.P. Kutter, Electrophoresis 25 (2004) 3498.

[5] M.E. Johnson, J.P. Landers, Electrophoresis 25 (2004) 3513.

[6] N.A. Lacher, K.E. Garrison, R.S. Martin, S.M. Lunte, Electrophoresis 22 (2001) 2526.

[7] A.T. Woolley, K. Lao, A.N. Glazer, R.A. Mathies, Anal. Chem. 70 (1998) 684.

[8] A. Hilmi, J.H.T. Luong, Anal. Chem. 72 (2000) 4677.

[9] M.A. Schwarz, B. Galliker, K. Fluri, T. Kappes, P.C. Hauser, Analyst 126 (2001) 147.

[10] X. Huang, R.N. Zare, S. Sloss, A.E. Ewing, Anal. Chem. 63 (1991) 189.

[11] R.M. Guijt, E. Baltussen, G. van der Steen, R.B.M. Schasfoort, S. Schlautmann, H.A.H. Billiet, J. Frank, G.W.K. van Dedem, A. van den Berg, Electrophoresis 22 (2001) 235.

[12] M.L. Chabinyc, D.T. Chiu, J.C. McDonald, A.D. Stroock, J.F. Christian, A.M. Karger, G.M. Whitesides, Anal. Chem. 73 (2001) 4491.

[13] K. Hata, Y. Kichise, T. Kaneta, T. Imasaka, Anal. Chem. 75 (2003) 1765.

[14] A.A. Grace, C.R. Gerfen, G. Aston-Jones, Adv. Pharmacol. 42 (1998) 655.

[15] R. Laverty, Drugs 16 (1978) 418.

[16] D. Arráez Román, A. Segura Carretero, C. Cruces Blanco, A. Fernández Gutiérrez, Biomed. Chromatogr. 18 (2004) 422.

[17] S. Parrot, V. Sauvinet, V. Riban, A. Depaulis, B. Renaud, L. Denoroy, J. Neurosci. Methods 140 (2004) 29. 
[18] S. Xiong, H. Han, R. Zhao, Y. Chen, G. Liu, Biomed. Chromatogr. 15 (2001) 83.

[19] A. Manz, D.J. Harrison, E.M.J. Verpoorte, J.C. Fettinger, A. Paulus, H. Lüdi, H.M. Widmer, J. Chromatogr. 593 (1992) 253.

[20] H.-F. Li, J.-M. Lin, R.-G. Su, K. Uchiyama, T. Hobo, Electrophoresis 25 (2004) 1907.
[21] N. Chiem, D.J. Harrison, Anal. Chem. 69 (1997) 373.

[22] D.J. Harrison, K. Fluri, K. Seiler, Z.H. Fan, C.S. Effenhauser, A. Manz, Science 261 (1993) 895.

[23] W.Z. Lu, R.M. Cassidy, Anal. Chem. 66 (1994) 200.

[24] F.-M. Matysik, J. Chromatogr. A 742 (1996) 229. 